Check for updates

Cite this: RSC Adv., 2017, 7, 20422

Received 10th December 2016 Accepted 3rd April 2017

DOI: 10.1039/c6ra28010b

rsc.li/rsc-advances

\title{
Autonomous self-healing multiwalled carbon nanotube nanocomposites with piezoresistive effect
}

\author{
Tongfei Wu (D) and Biqiong Chen (D)*
}

\begin{abstract}
Autonomous self-healing fatty acid rubber nanocomposites reinforced by multiwalled carbon nanotubes (MWCNTs) were synthesized by solution blending. The dispersion of MWCNTs in the self-healing fatty acid rubber matrix was observed by scanning electron microscopy. The reinforcement of MWCNTs on the mechanical properties and the self-healing capability of the self-healing fatty acid rubber/MWCNT nanocomposites were investigated by tensile testing. The effect of MWCNT concentration on the electrical conductivity of self-healing fatty acid rubber was studied and the electrically conductive behaviors of self-healing fatty acid rubber/MWCNT nanocomposites were interpreted using the percolation theory and tunneling model. The piezoresistive effect was observed in the nanocomposite with 19.7 vol\% MWCNTs, which demonstrates potential for pressure sensing applications.
\end{abstract}

\section{Introduction}

Inspired by nature, self-healing chemistry is attracting significant interest in the field of electronics. ${ }^{1}$ The capability to reconstruct the functionality after rupture will offer a significant advancement towards fabricating safer, longer-lasting and novel electronics. Technically, the recovery of functionalities can either be fully autonomous, or requires external assistance such as heat, light or chemicals to initiate the recovery process. ${ }^{2}$ There have been several approaches explored towards building autonomous self-repairing electronics, such as capsulereleasing techniques, ${ }^{3,4}$ self-healing rubber/conducting filler hybrids, ${ }^{5-9}$ and conducting polymer doughs. ${ }^{10}$ In comparison with capsule-releasing techniques, where self-healing cannot take place after capsules are exhausted, the electronics based on self-healing rubbers and polymer doughs both demonstrate that self-healing can act multiple times, providing repeatable recovery of the functionality. Owing to the lack of resilience in polymer doughs, self-healing rubbers are more desirable for flexible/stretchable electronics. The self-healing capability in self-healing rubbers relies on the reversible dynamic bonds of which the association or dissociation can occur spontaneously at ambient conditions, allowing the repair of damages at the molecular level to restore the properties of the original materials. ${ }^{11} \mathrm{~A}$ number of dynamic reversible bonds have been studied, including hydrogen bond, ${ }^{12-14}$ metal-ligand interaction, ${ }^{15} \pi-\pi$ stacking, ${ }^{16}$ molecular interdiffusion, ${ }^{17}$ and boron/ oxygen dative bond. ${ }^{18}$

Department of Materials Science and Engineering, University of Sheffield, Mappin Street, Sheffield S1 3JD, UK. E-mail: biqiong.chen@sheffield.ac.uk
Self-healing fatty acid rubber is a type of viscoelastic supramolecular polymers made from fatty acids, diethylenetriamine (DETA) and urea, first reported by Leibler and coworkers. ${ }^{14}$ The dynamically cross-linked supramolecular network is formed of a large number of di-/tri-functional building blocks with various strongly hydrogen-bonding amide groups. The glass transition temperature $\left(T_{\mathrm{g}}\right)$ of this kind of material is generally lower than room temperature (typically $10-25{ }^{\circ} \mathrm{C}$ ) ${ }^{19}$ allowing the association or dissociation of hydrogen bonds to occur spontaneously at ambient conditions and leading to autonomous self-healing behaviors. ${ }^{19}$ Owing to the excellent property of self-healing fatty acid rubber, it has stimulated increasing attempts in fabricating self-healing electronics such as high-energy lithium-ion batteries, ${ }^{20,21}$ thermal sensors, ${ }^{8}$ supercapacitors, ${ }^{22}$ and electronic skins. ${ }^{5,9}$

Carbon nanotubes (CNTs) have been explored as a promising nanofiller for conducting polymer nanocomposites, due to their high electrical conductivity ( $\sim 106 \mathrm{~S} \mathrm{~m}^{-1}$, metallic type), outstanding mechanical properties (1.5-4.8 GPa in strength), high flexibility, low density, and large aspect ratio (3001000). ${ }^{23-26}$ Theoretically, the large aspect ratio of CNTs enables significant enhancements of electrically conducting properties occurring with a very low percolation threshold for the formation of conducting networks. ${ }^{27,28}$ It has been found that the electrical percolation threshold in CNT nanocomposites varies between $0.001 \mathrm{vol} \%$ and $1 \mathrm{vol} \%$, depending on the nature of CNTs and polymer used, the dispersion of CNTs in polymer matrix, as well as the fabrication process (e.g., solution blending, melt blending, or in situ polymerization). ${ }^{23,29}$ Previous studies suggest that the tunneling resistance plays a dominant role in the electrical conductivity of polymer/CNT nanocomposites. ${ }^{30}$ And the tunneling resistance is responsive to 
various external factors. Numerous sensors based on tunneling resistances have been developed utilizing polymer/CNT nanocomposites, including strain sensors, ${ }^{31}$ infrared sensors, ${ }^{32}$ gas sensors, ${ }^{33}$ humidity sensor, ${ }^{34}$ and corrosion sensors. ${ }^{35}$

In this work, we prepared self-healing fatty acid rubber/ MWCNT nanocomposites with piezoresistive effect by solution blending for pressure sensing applications. The dispersion of MWCNTs in the matrix was observed using a scanning electron microscope. The effects of MWCNTs on mechanical properties and self-healing performance of self-healing fatty acid rubber were examined in depth by tensile tests. Electrically conducting behaviors of self-healing fatty acid rubber/MWCNT nanocomposites were studied using the percolation theory and tunneling model. The piezoresistivity and pressure sensing performance of a selected nanocomposite were investigated.

\section{Experimental}

\section{Materials}

Pripol 1017 produced from vegetable oils (75-80 wt\% dimer acids, $18-22 \mathrm{wt} \%$ trimer acids and $1-3 \mathrm{wt} \%$ monoacids $)^{2,19}$ was provided by Croda International Plc. Multiwalled carbon nanotubes (outer diameter: 10-15 nm, inner diameter: 2-6 nm and length: 0.1-10 $\mu \mathrm{m}$ ), diethylenetriamine (DETA, 99\%), urea ( $>99.0 \%)$, isopropanol ( $\geq 99.7 \%)$ and chloroform $(\geq 99.0 \%)$ were purchased from Sigma-Aldrich and used as received.

\section{Preparation of self-healing fatty acid rubber solution}

Self-healing fatty acid rubber was synthesized from Pripol 1017, DETA and urea following Leibler's method., ${ }^{\mathbf{8 1 4 , 1 9}}$ Pripol 1017 (46 g) and DETA (16 g) were mechanically stirred continuously at $160{ }^{\circ} \mathrm{C}$ for $24 \mathrm{~h}$ under a nitrogen atmosphere. The precursor was then dissolved in chloroform $(150 \mathrm{~mL})$, followed by washing with a solvent mixture containing $150 \mathrm{~mL}$ water and $35 \mathrm{~mL}$ methanol for three times using a separatory funnel to remove small-molecule derivatives. Urea (3.5 g) was added into the washed chloroform solution and chloroform was subsequently removed from the system by distillation with an oil bath at $135{ }^{\circ} \mathrm{C}$. The resulting compound was allowed to react at $135{ }^{\circ} \mathrm{C}$ for $3 \mathrm{~h}$ and then dissolved in isopropanol $(300 \mathrm{~mL})$ at $70{ }^{\circ} \mathrm{C}$ under mechanical stirring in a nitrogen atmosphere. The concentration of the final self-healing fatty acid rubber in isopropanol was $21.3 \mathrm{wt} \%$.

\section{Preparation of self-healing fatty acid rubber/MWCNT nanocomposites}

Self-healing fatty acid rubber/MWCNT nanocomposites were prepared by solution blending. MWCNTs ( $2 \mathrm{~g}$ ) were added into $300 \mathrm{~mL}$ isopropanol. A Silverson L5M-A shear mixer was employed for MWCNT exfoliation and debundling. The mixture was stirred at $9500 \mathrm{rpm}$ for $20 \mathrm{~min}$ within an ice bath to decelerate the evaporation of isopropanol. The solid content of the resultant MWCNT suspension was measured as $0.583 \mathrm{wt} \%$ and used for preparing nanocomposites. A desired amount of MWCNT isopropanol suspension was weighed and mixed with self-healing fatty acid rubber solution under magnetically stirring for $2 \mathrm{~h}$. The resulted mixture was poured into a polytetrafluoroethylene (PTFE) dish and kept in a fume cupboard for $24 \mathrm{~h}$ to allow the evaporation of most isopropanol. The residual solvent was removed in vacuum at $60{ }^{\circ} \mathrm{C}$ for $24 \mathrm{~h}$. Samples with $0,0.02,0.04,0.08,0.16$, and 0.32 mass fractions of MWCNTs in self-healing fatty acid rubber nanocomposites were prepared by this method. The corresponding nominal volume fractions of MWCNTs were 0, 0.0105, 0.0212, 0.0433, 0.0901 and 0.197 , respectively, based on densities of self-healing fatty acid rubber (experimentally measured as $0.905 \mathrm{~g} \mathrm{~cm}^{-3}$ ) and MWCTs $\left(1.74 \mathrm{~g} \mathrm{~cm}^{-3}\right) .{ }^{36}$

\section{Characterization}

Attenuated total reflectance-Fourier transform infrared (ATRFTIR) spectroscopy was carried out on a Frontier Optica spectrophotometer (PerkinElmer) in the wavenumber region between 4000 to $600 \mathrm{~cm}^{-1}$ with a resolution of $1 \mathrm{~cm}^{-1}$. To extract the MWCNTs from the nanocomposite, the fatty acid rubber in the nanocomposite containing 19.7 vol\% MWCNTs was dissolved in isopropanol and the MWCNTs were collected by centrifugation. The dissolving-centrifugation cycles had been repeated three times before the separated MWCNTs were dried for FTIR. The microstructure of MWCNTs and the fracture surface of self-healing fatty acid rubber/MWCNT nanocomposite were investigated by scanning electron microscopy (SEM) (Inspect F, FEI) using a $10 \mathrm{keV}$ acceleration voltage. For cross-sectional SEM imaging, the sample was fractured in liquid nitrogen prior to SEM observations. Tensile tests were carried out at room temperature $\left(25^{\circ} \mathrm{C}\right)$ using a Lloyd universal testing machine (Ametek Inc.) with a $10 \mathrm{~N}$ load cell and a $100 \mathrm{~mm}$ $\min ^{-1}$ testing speed. For each sample, five specimens were tested. The electrical properties of the sample were monitored in two-point via a benchtop multimeter (Agilent 34401A, Keysight Technologies Inc.). For the conductivity test, a cylindrical sample $(\Phi 10 \times 5 \mathrm{~mm})$ was used and contacted to the circuit via two plate aluminium electrodes. For the piezoresistivity measurement, the resistance of the sample was recorded under various external pressures applied by the Lloyd universal testing machine. Differential scanning calorimetry (DSC) was performed on a Diamond DSC (Perkin-Elmer) at a heating/cooling rate of $10{ }^{\circ} \mathrm{C} \mathrm{min}^{-1}$ in a nitrogen atmosphere. The second heating was used to determine the glass transition temperature of self-healing fatty acid rubber in different nanocomposites to avoid the effect of heating history.

\section{Results and discussion}

\section{Characterization of as-synthesized self-healing fatty acid rubber and its MWCNT nanocomposites}

Self-healing fatty acid rubber was synthesized through two steps as shown in Scheme 1a: (i) the condensation between dimer/ trimer fatty acids and diethylene triamine (DETA), and subsequently (ii) the transamidation of the resulted oligoamidoamines (the precursor) with urea, leading to the extension of the molecular chains, introducing chemical cross-links and intensifying strongly hydrogen bonding points consisting of amide 
(a)

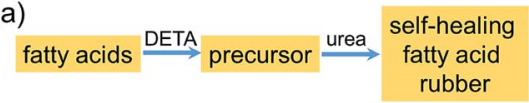

(b)
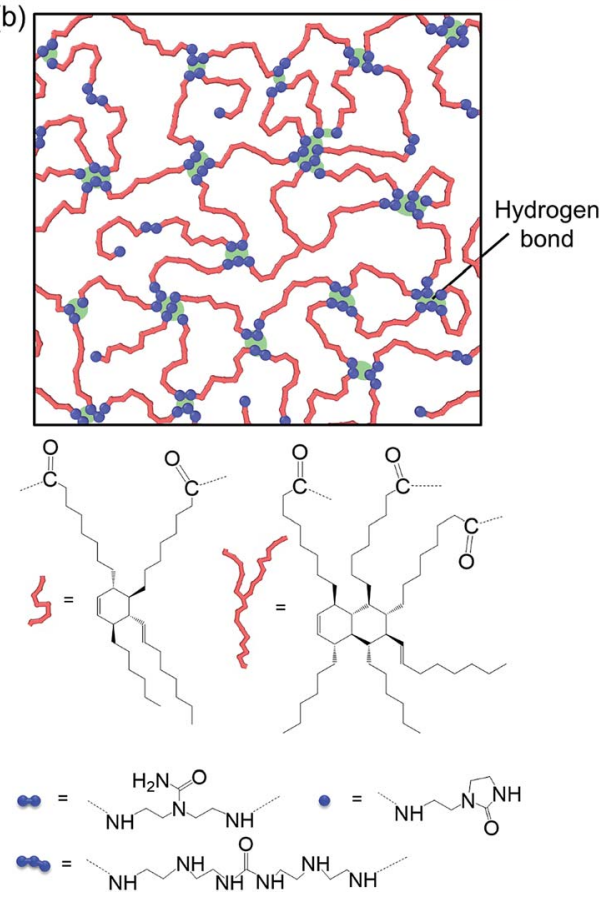

Scheme 1 (a) The synthesis of self-healing fatty acid rubber and (b) its representative chemical structure rich of hydrogen bonds.

groups (Scheme 1b). ${ }^{14}$ It was noted that a high density of chemical cross-links would benefit the mechanical properties of products, but reduce their solubility. Therefore, the density of chemical cross-links should be controlled by limiting the reaction time period in the second step to ease the dissolving process. In this work, we chose three hours for the second-step reaction and obtained a clear isopropanol solution (inset of Fig. 1a). The glass transition temperature $\left(T_{\mathrm{g}}\right)$ of the product dried from the isopropanol solution was $11.6^{\circ} \mathrm{C}$ determined by DSC, which is reliable in comparison with $T_{\mathrm{g}}$ values (typically $10-25{ }^{\circ} \mathrm{C}$ ) of self-healing fatty acid rubber reported in previous work. ${ }^{19}$ The chemical structure was characterized by FTIR in Fig. 1a. The FTIR spectrum shows a number of vibrations: 3285 $\mathrm{cm}^{-1}, 3202 \mathrm{~cm}^{-1}, 3078 \mathrm{~cm}^{-1}$ and $1543 \mathrm{~cm}^{-1}$ (N-H stretching), $2918 \mathrm{~cm}^{-1}$ and $2848 \mathrm{~cm}^{-1}$ (C-H stretching), $1647 \mathrm{~cm}^{-1}$ (C=O stretching in amide), and $1608 \mathrm{~cm}^{-1}(\mathrm{C}=\mathrm{O}$ stretching in urea), which are in excellent agreement with characteristic bands of self-healing fatty acid rubber in the literature. ${ }^{37}$ The assynthesized product appears glassy and resilient, as shown in Fig. 1b. Its autonomous self-healing capability will be discussed below. The deformation hysteresis of the product was measured by cyclic tensile tests (Fig. 1c). Deformation velocity was set to $100 \mathrm{~mm} \mathrm{~min}{ }^{-1}$ and maximum strain was set to $100 \%$ of the original length of the sample (the inset of Fig. 1c). The result indicates that the product exhibited a significantly large deformation hysteresis, suggesting a viscoelastic characteristic. ${ }^{14}$ The hysteresis energy (i.e. the ratio of the area between the first loading and unloading curves (1) and (2) to the area under

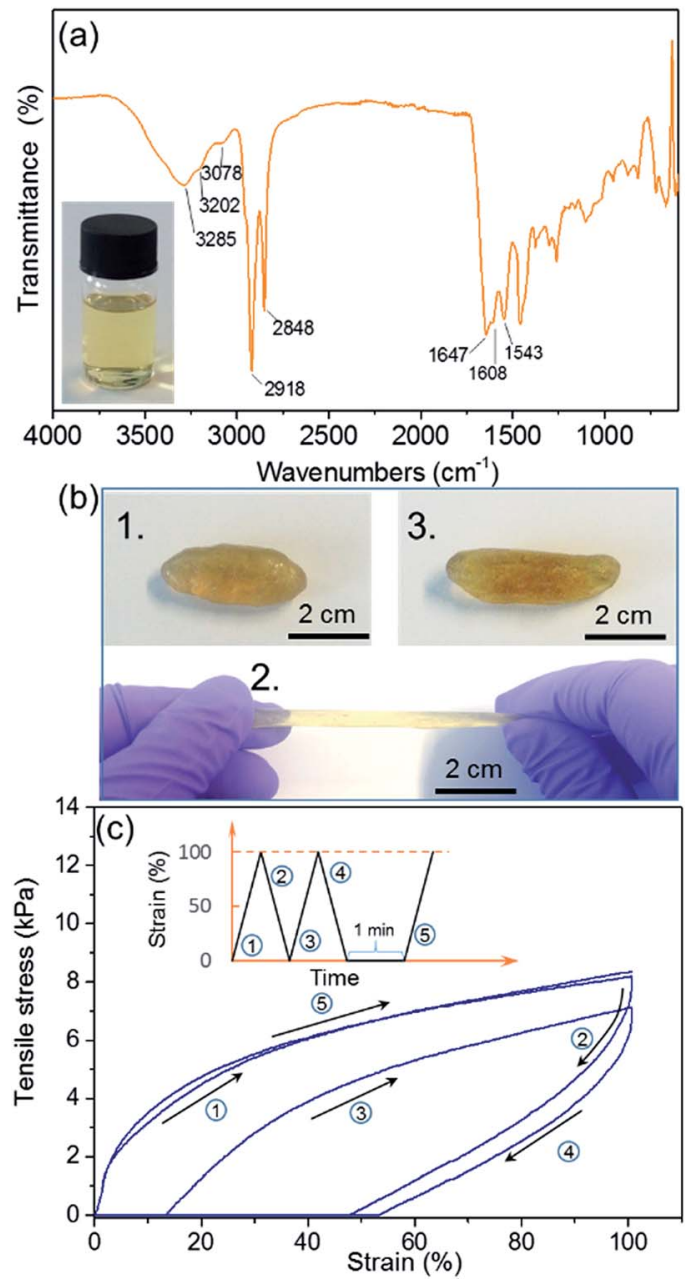

Fig. 1 (a) FTIR spectrum of the as-synthesized self-healing fatty acid rubber product. Inset of (a): photograph of its isopropanol solution. (b) Photographs of the product demonstrating the resilience: (1) original, (2) stretched and (3) released. (c) Cyclic tensile stress-strain curves showing the resilience of the product. Numbers (1)-(5) indicate the sequence of loading and unloading curves as depicted in inset of (c).

curve (1)) was calculated as $75.3 \%$. The residual strain reached $0 \%$ (the original point of loading curve (5) after 1 min relaxation at stress-free state, suggesting a full recovery in the product after a delayed returning.

To investigate the influence of MWCNTs (Fig. 2a) as an electrically conductive and mechanically reinforcing nanofiller within the self-healing fatty acid rubber, we prepared five selfhealing fatty acid rubber/MWCNT nanocomposites with different concentrations of MWCNTs (from 1.05 to 19.7 vol\%) by solution blending. Cross-sectional SEM image of the selfhealing fatty acid rubber/MWCNT nanocomposite with the highest MWCNT content $(19.7 \mathrm{vol} \%)$ still demonstrates a homogenous dispersion of MWCNTs in the self-healing fatty acid rubber matrix and a good wettability of self-healing fatty acid rubber on the surface of MWCNTs, as shown in Fig. 2b. This might be related to the nonpolar chemical structure of the fatty acid rubber that contributes to the compatibility, as well as its viscoelastic properties. The interactions between the 

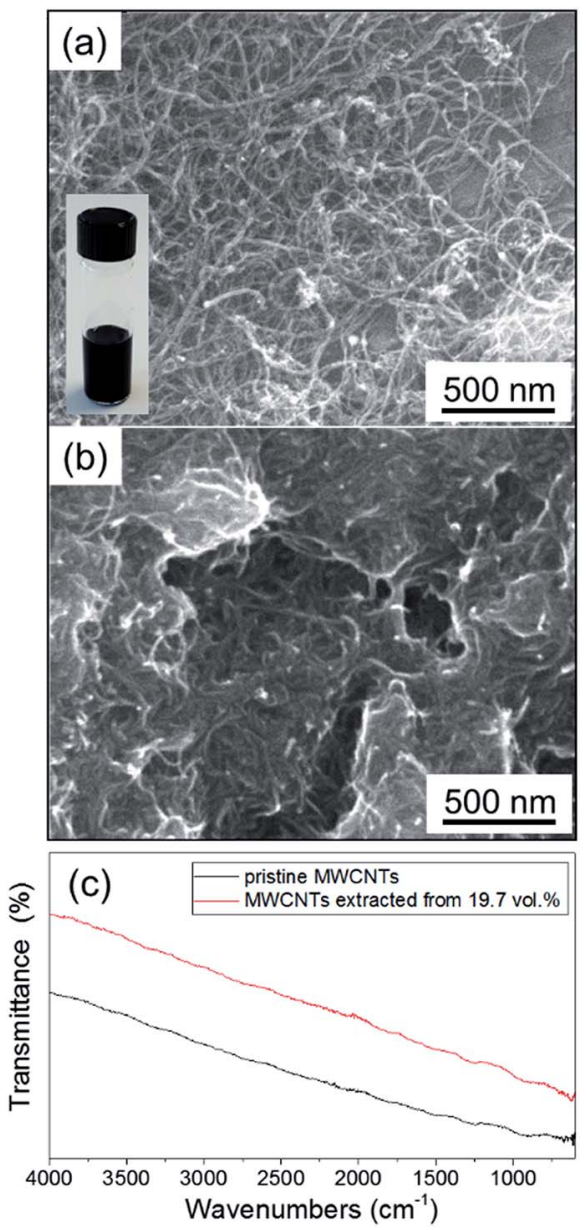

Fig. 2 SEM images of (a) pristine MWCNTs and (b) self-healing fatty acid rubber/MWCNT nanocomposite with 19.7 vol\% MWCNTs. Inset of (a): photograph of the MWCNT isopropanol suspension. (c) FTIR spectra of pristine MWCNTs and MWCNTs extracted from the nanocomposite.

MWCNTs and the fatty acid rubber are physical, as confirmed by FTIR. As shown in Fig. 2c, the MWCNTs separated from the nanocomposite with 19.7 vol\% MWCNTs exhibit similar vibration peaks to those of pristine MWCNTs, without the presence of the characteristic absorption peaks of fatty acid rubber.

\section{Mechanical properties of self-healing fatty acid rubber/ MWCNT nanocomposites}

The reinforcement of MWCNTs on the mechanical properties of self-healing fatty acid rubber was determined by tensile tests. The results are summarized in Fig. 3. The tensile strength, elongation at break, and Young's modulus were $64.8 \mathrm{kPa}, 910 \%$, and $35.5 \mathrm{kPa}$ for neat rubber, respectively. The tensile strength increased with MWCNT concentration. The incorporation of MWCNTs raised the tensile strength up to $596 \mathrm{kPa}$ with a MWCNT concentration of 19.7 vol\% (corresponding to an 8time increment), suggesting the efficient stress transfer in the nanocomposite. The elongation at break of self-healing fatty acid rubber/MWCNT nanocomposites decreased dramatically when increasing the concentration of MWCNTs. For instance,
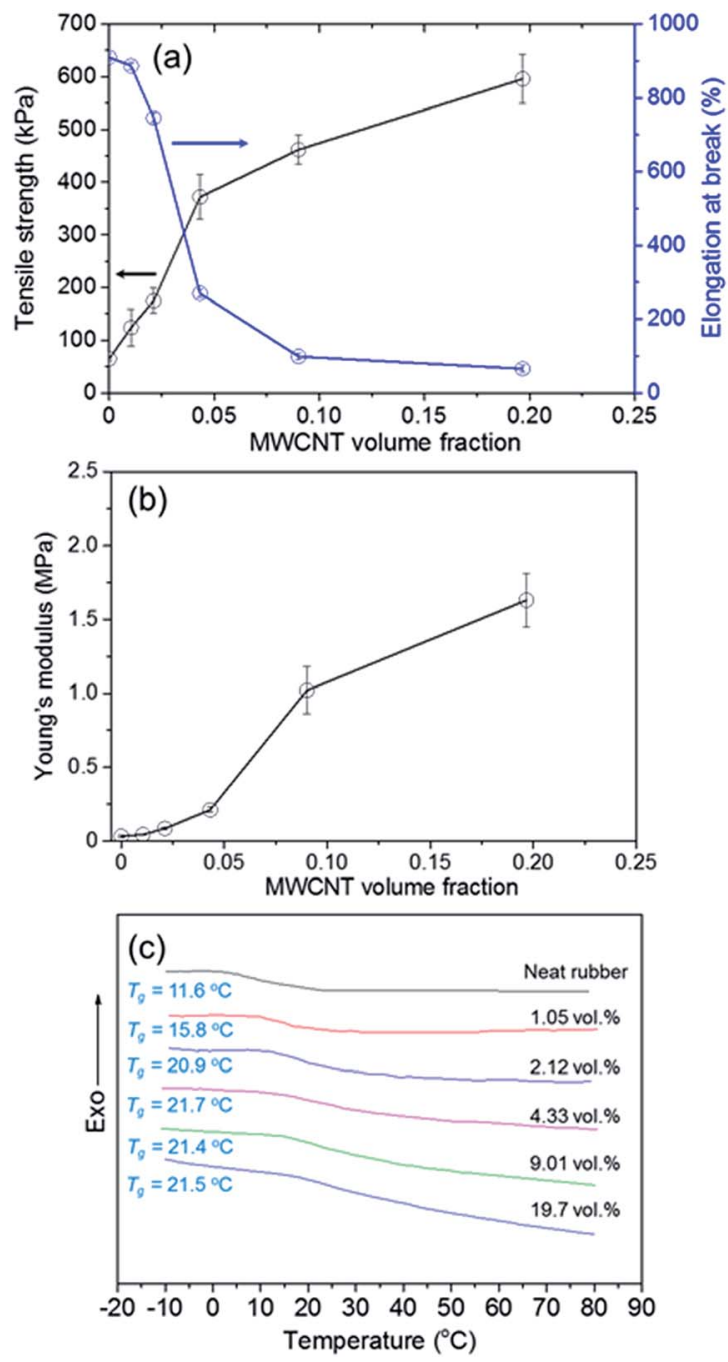

Fig. 3 (a) Tensile strength and elongation at break and (b) Young's modulus of self-healing fatty acid rubber/MWCNT nanocomposites as a function of MWCNT concentration. (c) Glass transition temperature $\left(T_{\mathrm{g}}\right)$ of self-healing fatty acid rubber in different nanocomposites measured by DSC during the second heating at a scan rate of $10^{\circ} \mathrm{C}$ $\min ^{-1}$.

the elongation at break was $270 \%$ with 4.33 vol\% MWCNTs and 65.6\% with 19.7 vol\% MWCNTs. This can be attributed to the confinement effect of rigid nanofillers on the mobility of polymer chains, ${ }^{38}$ which was confirmed by the measurement of $T_{\mathrm{g}}$ of self-healing fatty acid rubber in each nanocomposite (Fig. 3c). It can be seen that $T_{\mathrm{g}}$ has an upward trend with increasing MWCNT concentration, from $11.6{ }^{\circ} \mathrm{C}$ for neat rubber up to $21.7{ }^{\circ} \mathrm{C}$ with $4.33 \mathrm{vol} \%$ MWCNTs. This large $T_{\mathrm{g}}$ shift $\left(\sim 10^{\circ} \mathrm{C}\right)$ suggests the efficient suppression of the mobility of self-healing fatty acid rubber chains by MWCNTs. That is attributable to the large specific surface area of MWCNTs ${ }^{39}$ and good wettability of self-healing fatty acid rubber on the surface of MWCNTs. Young's modulus of self-healing fatty acid rubber/MWCNT nanocomposites increased significantly with the content of MWCNTs. The improvement was 5.89 times with 4.33 vol\% MWCNTs, 32.6 times with 9.01 vol\% MWCNTs, and 52.3 times 
with 19.7 vol\% MWCNTs, in comparison with that for neat rubber. These reinforcements are ascribed to the excellent physical and mechanical properties of MWCNTs as well as a good dispersion of MWCNTs in the rubber matrix. ${ }^{40}$

A key feature of self-healing fatty acid rubber is its autonomous, room-temperature (RT, $25{ }^{\circ} \mathrm{C}$ ) mechanically self-healing property. It means, when self-healing fatty acid rubber suffers cracks or fractures, simply bringing the broken pieces together will lead to spontaneous healing. Fig. 4a demonstrates the mechanically self-healing behavior of the MWCNT-reinforced self-healing fatty acid rubber nanocomposite (19.7 vol\%). According to the literature, ${ }^{5}$ the mechanical healing efficiency can be calculated as the ratio of the restored toughness (the area under the stress-strain curve) to the original toughness. Fig. $4 \mathrm{~b}$ compares the typical tensile stress-strain curves of samples healed for $5 \mathrm{~min}$ at two different temperatures (RT and $50{ }^{\circ} \mathrm{C}$ ) and their healing efficiencies are summarized in Fig. 4c. The toughness of neat rubber was able to almost fully recover $(99.5 \%)$ after $5 \mathrm{~min}$ of contact at RT. For self-healing fatty acid rubber/MWCNT nanocomposites, the self-healing capability decreased dramatically with the MWCNT concentration. For

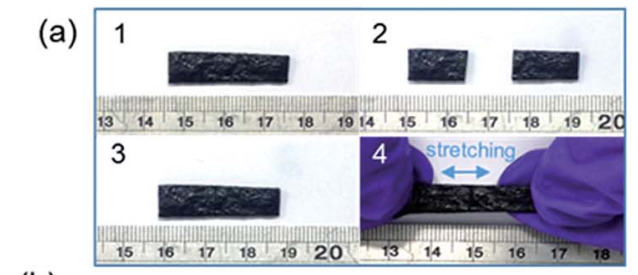

(b) 700

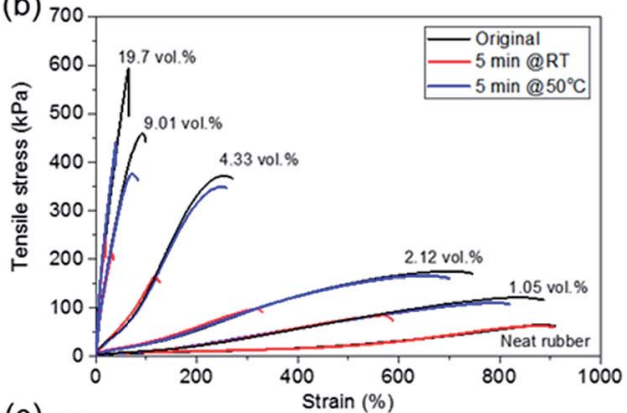

(c)

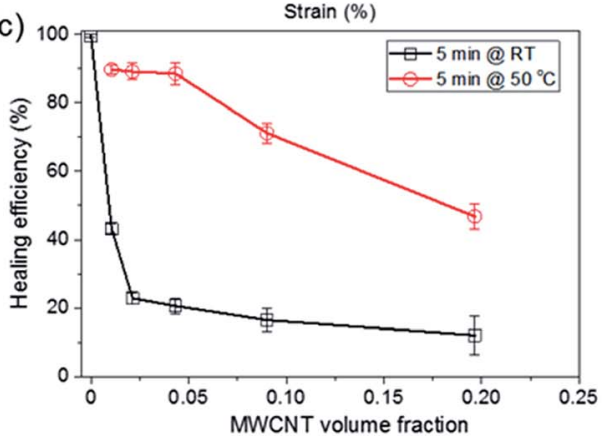

Fig. 4 (a) Photographs of the self-healing fatty acid rubber/MWCNT nanocomposite with 19.7 vol\% MWCNTs demonstrating the autonomous healing property at room temperature (RT, $\left.25^{\circ} \mathrm{C}\right)$ : (1) original; (2) completely severed; (3) contacted for healing; and (4) healed after 5 min of contact. (b) Typical tensile stress-strain curves of original samples and samples healed for $5 \mathrm{~min}$ at RT and $50^{\circ} \mathrm{C}$. (c) Self-healing efficiency of samples at two different temperatures. instance, the healing efficiency was determined as $43.2 \%$ with 1.05 vol\% MWCNTs after 5 min of contact at RT and $12.1 \%$ with 19.7 vol\% MWCNTs. The decreasing self-healing capability with increasing MWCNT amount can be attributed to the fact that MWCNTs could form a rigid network hindering the migration of self-healing fatty acid rubber for the contact through confining polymer chains. ${ }^{38}$ Hence, the healing efficiency can be improved by heating, because an elevated temperature facilitates the mobility of polymer chains. In comparison with the performance at RT, the self-healing capability at $50{ }^{\circ} \mathrm{C}$ was improved to $89.7 \%, 89.1 \%, 88.5 \%, 71.2 \%$ and $46.8 \%$ for the selfhealing fatty acid rubber/MWCNT nanocomposite with 1.05, 2.12, 4.33, 9.01 and 19.7 vol\% MWCNTs, respectively.

\section{Electrically conducting behaviors of self-healing fatty acid rubber/MWCNT nanocomposites}

The MWCNT network provides a continuous conducting network in the insulating rubber matrix. The experimentally determined electrical conductivity $(\sigma)$ increases with the MWCNT concentration, from $1.53 \times 10^{-5} \mathrm{~S} \mathrm{~cm}^{-1}$ with 1.05 vol\% MWCNTs to $5.01 \times 10^{-3} \mathrm{~S} \mathrm{~cm}^{-1}$ with 19.7 vol\% MWCNTs (Fig. 5a). The conductivity data were interpreted by the percolation theory and tunneling model. ${ }^{\mathbf{4 1 , 4 2}}$ In classical percolation theory, the dependence of $\sigma$ values of the composites on the conductive filler concentration $(p)$ above the percolation threshold $\left(p_{c}\right)$ can be described by a scaling law as shown in eqn (1). ${ }^{43}$

$$
\sigma=\sigma_{0}\left(p-p_{\mathrm{c}}\right)^{t} \text { for } p>p_{\mathrm{c}}
$$

here $\sigma_{0}$ is a fitted conductive constant and $t$ is the critical exponent. A non-linear curve fitting of the experimental data was performed in order to obtain the best fitted values of constants in eqn (1). $t$ was determined as 1.67, in fair agreement with the value of 1.6-2.0 obtained by Monte Carlo studies in three dimensions. ${ }^{44}$ The value of $p_{\mathrm{c}}$ was $4.53 \times 10^{-3}$ (i.e. 0.453 vol\%) and $0.084 \mathrm{~S} \mathrm{~cm}^{-1}$ for $\sigma_{0}$. The value of $\sigma_{0}$ should approach the conductivity of the MWCNT cluster itself (typically 1-5 S $\mathrm{cm}^{-1}$ under 0.1-5 MPa as reported in the literature ${ }^{45}$ ). However, the fitted $\sigma_{0}$ was much lower than those values. This might be caused by the presence of the contact resistance between the clusters of MWCNTs in the nanocomposites, which was formed by the thin insulating rubber layer on MWCNTs and significantly reduced the effective conductivity of the MWCNT network. ${ }^{46}$ That means the electrons needed more energy to tunnel from one conductive cluster to another separated by the thin insulating layer.

According to the thermal fluctuation-induced tunneling model, ${ }^{47}$ the electrical conductivity for a homogeneous conductive filler/insulating polymer composite can be described by the behavior of a single tunnel junction at a given temperature, and a linear relation between $\ln \sigma$ and $p^{-1 / 3}$ is expected, namely $\ln \sigma$ $\propto p^{-1 / 3}$. From the inset of Fig. $5 \mathrm{a}$, it can be seen that the electrical conduction in self-healing fatty acid rubber/MWCNT nanocomposites followed well the tunneling model. Therefore, the low value of $\sigma_{0}$ suggests a good wettability of self-healing fatty acid rubber on the surface of MWCNTs, which is in accordance with 

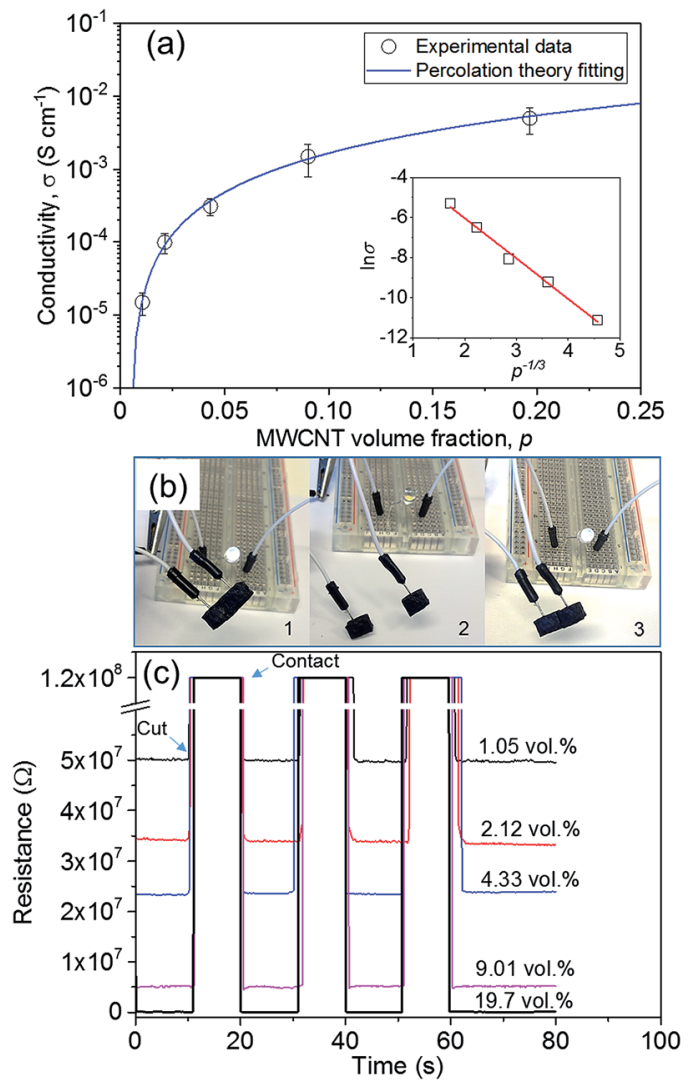

Fig. 5 (a) Electrical conductivity of self-healing fatty acid rubber/ MWCNT nanocomposites versus the MWCNT concentration. Inset of (a): plot of $\ln \sigma$ vs. $p^{-1 / 3}$. (b) Demonstration of the self-repairing property of the self-healing fatty acid rubber/MWCNT nanocomposite (19.7 vol\%) in an LED circuit: (1) original; (2) completely severed; (3) healed by applying a gentle force $(\sim 1 \mathrm{kPa})$ for $\sim 1 \mathrm{~s}$. (c) Resistance versus time during the electrically healing process.

the observation of SEM. Fig. 5b demonstrates the self-repairing behavior of an electronic circuit containing the self-healing nanocomposite. The change in resistance during the repeated self-healing processes is shown in Fig. $5 \mathrm{c}$. It can be seen that the resistance immediately dropped to nearly its original value, as soon as the two fracture surfaces were gently brought together. The conductive healing efficiency is determined as the ratio of the original resistance to the restored resistance. The healing efficiency is calculated as higher than $98.2 \%$ for all self-healing fatty acid rubber/MWCNT nanocomposites.

Based on the tunneling model, the compression-induced reduction in the thickness of insulating polymer layer would raise the electrical conductivity, leading to the piezoresistive effect. $^{48}$ The piezoresistive effect in self-healing fatty acid rubber/MWCNT nanocomposite with 19.7 vol\% MWCNTs was investigated (Fig. 6a). The device setup with a sandwich configuration was shown in the inset of Fig. 6a. In the device, the middle nanocomposite layer (rubber/MWCNT) with a thickness of $100 \mu \mathrm{m}$ was formed by compression and selfadhered to the two Al conductors. As anticipated, the resistance decreased with increasing pressure, but exhibited a nonlinear relation in the range from zero pressure up to $400 \mathrm{kPa}$.
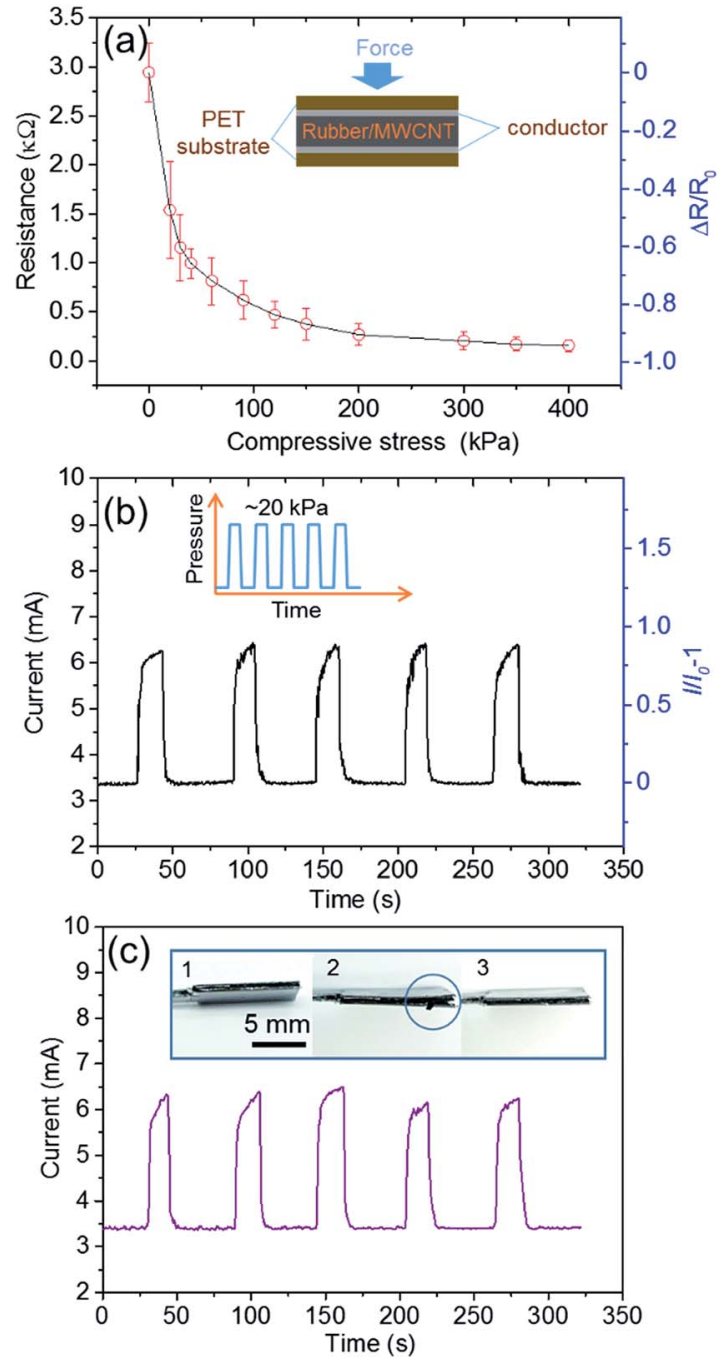

Fig. 6 (a) Resistance $(R)$ versus pressure for the self-healing fatty acid rubber/MWCNT nanocomposite with 19.7 vol\% MWCNTs. The inset of (b) is the schematic setup for the pressure-responsive test. (b) Current (I) versus time for the as-established device in response to pulse pressure application of approximately $20 \mathrm{kPa}$. The dynamic test was conducted at a bias voltage of $10 \mathrm{~V}$. Inset of (b): applied pressure against time. (c) Current versus time for the healed device after a damage demonstrated in the inset of (c): (1) original; (2) with a cut in the middle of rubber/MWCNT layer; (3) healed by applying a press ( $\sim 400 \mathrm{kPa})$ for $\sim 10 \mathrm{~s}$.

For instance, the resistance $\left(R_{0}\right)$ was $2.94 \times 10^{3} \Omega$ at zero pressure, $618 \Omega$ at $90 \mathrm{kPa}, 268 \Omega$ at $200 \mathrm{kPa}$, and $158 \Omega$ at $400 \mathrm{kPa}$, where the corresponding reduction factors $\left(\Delta R / R_{0}\right)$ were $-79.0 \%,-90.9 \%$ and $-94.6 \%$, respectively. Dynamic pressure sensitivity was monitored while measuring the current variations with the repeated application of $\sim 20 \mathrm{kPa}$ (Fig. 6b). Under a compression of $\sim 20 \mathrm{kPa}$, the measured current becomes noticeably higher (a sensitivity $\left(\Delta I / I_{0}-1\right)$ of $90 \%$ ); once the force was released, the current gradually decreased in 7-10 $\mathrm{s}$ to the initial value. These dynamic compression characteristics are comparable or superior to those of devices based on CNT/ polyurethane composites and CNT/vinyl ester composites. ${ }^{49,50}$ With these fast response and recovery times, the as-established 
pressure sensor exhibited a significant change in the intensity of current signal. The five testing cycles showing the same profiles suggested good repeatability in responses to dynamic loading. Fig. 6c demonstrates that the as-established sensor could be repaired by applying a press $(\sim 400 \mathrm{kPa})$ for $\sim 10 \mathrm{~s}$ after a damage (like a cutting) in the rubber/MWCNT layer, owning to the self-healing capability of rubber/MWCNT nanocomposites.

\section{Conclusions}

Autonomous self-healing conducting nanocomposites were synthesized by embedding MWCNTs in the self-healing matrix of fatty acid rubber. The tensile strength and Young's modulus of the self-healing fatty acid rubber were significantly improved by the addition of MWCNTs. However, the elongation at break was dramatically reduced, owing to the confinement effect of rigid nanofillers on the mobility of polymer chains. The nanocomposites demonstrated repetitive rapid mechanically selfhealing capability at ambient temperature where the healing efficiency could be further improved through increasing the mobility of polymer chains by heating. The electrically conducting behaviors of the nanocomposites followed the percolation theory and tunneling model. The nanocomposite with 19.7 vol\% MWCNTs exhibited a non-linear piezoresistive effect. In dynamic pressure sensing test, the nanocomposite exhibited a fast recovery time (7-10 s) and good repeatability, while a sensitivity of $90 \%$ was achieved at $\sim 20 \mathrm{kPa}$. The sensor made up of the nanocomposite could be repaired by applying a press after a damage, owning to its self-healing capability.

\section{Acknowledgements}

This project is supported by the European Commission's Horizon 2020 research and innovation programme under the Marie Skłodowska-Curie grant agreement No. 656467.

\section{Notes and references}

1 S. J. Benight, C. Wang, J. B. H. Tok and Z. Bao, Prog. Polym. Sci., 2013, 38, 1961-1977.

2 B. J. Blaiszik, S. L. B. Kramer, S. C. Olugebefola, J. S. Moore, N. R. Sottos and S. R. White, Annu. Rev. Mater. Res., 2010, 40, 179-211.

3 E. Palleau, S. Reece, S. C. Desai, M. E. Smith and M. D. Dickey, Adv. Mater., 2013, 25, 1589-1592.

4 B. J. Blaiszik, S. L. B. Kramer, M. E. Grady, D. A. McIlroy, J. S. Moore, N. R. Sottos and S. R. White, Adv. Mater., 2012, 24, 398-401.

5 B. C. K. Tee, C. Wang, R. Allen and Z. Bao, Nat. Nanotechnol., 2012, 7, 825-832.

6 H. Sun, X. You, Y. Jiang, G. Guan, X. Fang, J. Deng, P. Chen, Y. Luo and H. Peng, Angew. Chem., Int. Ed., 2014, 53, 95269531.

7 S. Michel, B. T. T. Chu, S. Grimm, F. A. Nuesch, A. Borgschulte and D. M. Opris, J. Mater. Chem., 2012, 22, 20736-20741.
8 Y. He, S. Liao, H. Jia, Y. Cao, Z. Wang and Y. Wang, Adv. Mater., 2015, 27, 4622-4627.

9 E. D'Elia, S. Barg, N. Ni, V. G. Rocha and E. Saiz, Adv. Mater., 2015, 27, 4788-4794.

10 J. Y. Oh, S. Kim, H. K. Baik and U. Jeong, Adv. Mater., 2016, 28, 4455-4461.

11 M. W. Keller, S. R. White and N. R. Sottos, Adv. Funct. Mater., 2007, 17, 2399-2404.

12 J. Hentschel, A. M. Kushner, J. Ziller and Z. Guan, Angew. Chem., Int. Ed., 2012, 51, 10561-10565.

13 Y. Chen, A. M. Kushner, G. A. Williams and Z. Guan, Nat. Chem., 2012, 4, 467-472.

14 P. Cordier, F. Tournilhac, C. Soulie-Ziakovic and L. Leibler, Nature, 2008, 451, 977-980.

15 D. Mozhdehi, S. Ayala, O. R. Cromwell and Z. Guan, J. Am. Chem. Soc., 2014, 136, 16128-16131.

16 S. Burattini, B. W. Greenland, D. H. Merino, W. Weng, J. Seppala, H. M. Colquhoun, W. Hayes, M. E. Mackay, I. W. Hamley and S. J. Rowan, J. Am. Chem. Soc., 2010, 132, 12051-12058.

17 M. A. Rahman, L. Sartore, F. Bignotti and L. Di Landro, ACS Appl. Mater. Interfaces, 2013, 5, 1494-1502.

18 A. Juhász, P. Tasnádi and L. Fábry, Phys. Educ., 1984, 19, 302.

19 D. Montarnal, F. Tournilhac, M. Hidalgo, J. L. Couturier and L. Leibler, J. Am. Chem. Soc., 2009, 131, 7966-7967.

20 C. Wang, H. Wu, Z. Chen, M. T. McDowell, Y. Cui and Z. Bao, Nat. Chem., 2013, 5, 1042-1048.

21 Y. Zhao, Y. Zhang, H. Sun, X. Dong, J. Cao, L. Wang, Y. Xu, J. Ren, Y. Hwang, I. H. Son, X. Huang, Y. Wang and H. Peng, Angew. Chem., Int. Ed., 2016, 55, 14384-14388.

22 H. Wang, B. Zhu, W. Jiang, Y. Yang, W. R. Leow, H. Wang and X. Chen, Adv. Mater., 2014, 26, 3638-3643.

23 M. Moniruzzaman and K. I. Winey, Macromolecules, 2006, 39, 5194-5205.

24 N. G. Sahoo, S. Rana, J. W. Cho, L. Li and S. H. Chan, Prog. Polym. Sci., 2010, 35, 837-867.

25 R. H. Baughman, A. A. Zakhidov and W. A. de Heer, Science, 2002, 297, 787-792.

26 J. N. Coleman, U. Khan and Y. K. Gun'ko, Adv. Mater., 2006, 18, 689-706.

27 Y. Wang, G. J. Weng, S. A. Meguid and A. M. Hamouda, J. Appl. Phys., 2014, 115, 193706.

28 C. Li and T. W. Chou, Appl. Phys. Lett., 2007, 90, 174108.

29 W. Bauhofer and J. Z. Kovacs, Compos. Sci. Technol., 2009, 69, 1486-1498.

30 C. Li, E. T. Thostenson and T. W. Chou, Appl. Phys. Lett., 2007, 91, 223114.

31 N. Hu, Y. Karube, C. Yan, Z. Masuda and H. Fukunaga, Acta Mater., 2008, 56, 2929-2936.

32 B. Pradhan, K. Setyowati, H. Liu, D. H. Waldeck and J. Chen, Nano Lett., 2008, 8, 1142-1146.

33 K. H. An, S. Y. Jeong, H. R. Hwang and Y. H. Lee, Adv. Mater., 2004, 16, 1005-1009.

34 K. P. Yoo, L. T. Lim, N. K. Min, M. J. Lee, C. J. Lee and C. W. Park, Sens. Actuators, B, 2010, 145, 120-125. 
35 J. L. Kenneth, K. Junhee, P. L. Jerome, K. Nadine Wong Shi and A. K. Nicholas, Smart Mater. Struct., 2007, 16, 429.

36 S. H. Kim, G. W. Mulholland and M. R. Zachariah, Carbon, 2009, 47, 1297-1302.

37 D. Montarnal, P. Cordier, C. Soulié-Ziakovic, F. Tournilhac and L. Leibler, J. Polym. Sci., Part A: Polym. Chem., 2008, 46, 7925-7936.

38 P. Rittigstein, R. D. Priestley, L. J. Broadbelt and J. M. Torkelson, Nat. Mater., 2007, 6, 278-282.

39 J. H. Lehman, M. Terrones, E. Mansfield, K. E. Hurst and V. Meunier, Carbon, 2011, 49, 2581-2602.

40 H. Xia and M. Song, Soft Matter, 2005, 1, 386-394.

41 D. Stauffer, Introduction to the percolation theory, Francis and Taylor, London, 2nd edn, 1991.

42 P. Sheng, E. K. Sichel and J. I. Gittleman, Phys. Rev. Lett., 1978, 40, 1197-1200.
43 M. Weber and M. R. Kamal, Polym. Compos., 1997, 18, 711725.

44 M. T. Connor, S. Roy, T. A. Ezquerra and F. J. Baltá Calleja, Phys. Rev. B: Condens. Matter Mater. Phys., 1998, 57, 22862294.

45 B. Marinho, M. Ghislandi, E. Tkalya, C. E. Koning and G. de With, Powder Technol., 2012, 221, 351-358.

46 Z. Ounaies, C. Park, K. E. Wise, E. J. Siochi and J. S. Harrison, Compos. Sci. Technol., 2003, 63, 1637-1646.

47 T. A. Ezquerra, M. Kulescza and F. J. Baltá-Calleja, Synth. Met., 1991, 41, 915-920.

48 L. Chen, G. H. Chen and L. Lu, Adv. Funct. Mater., 2007, 17, 898-904.

49 J. J. Ku-Herrera and F. Avilés, Carbon, 2012, 50, 25922598.

50 T. Zhai, D. Li, G. Fei and H. Xia, Composites, Part A, 2015, 72, 108-114. 